\title{
Comparative Performance of MANET Routing Protocols at varying Speed in Different Traffic Patterns
}

\author{
Harpal $^{1}$, Sandeep Kaushal ${ }^{2}$, Gursharan Singh ${ }^{3}$ \\ M.Tech Research Scholar, Department of Electronics and Communication Engineering, Amritsar College of \\ Engineering and Technology, Amritsar, India ${ }^{1,3}$ \\ Associate Professor, Department of Electronics and Communication Engineering, Amritsar College of \\ Engineering and Technology, Amritsar, India ${ }^{2}$
}

\begin{abstract}
This paper evaluates the performance of two different mobile ad-hoc networks (MANET) routing protocols based upon one of the Mobility Model: Manhattan Grid (MG) with varying Speed of the mobile nodes in two different traffic Patterns. We have considered CBR and an Exponential Traffic Pattern (which is based on Exponential distribution). Simulations have been carried out by using Network Simulator Version 2.35 (NS-2) and its associated tools for analysis of results. Comparative analysis of simulation results includes the value of Packet Delivery Ratio (PDR), Throughput and Normalized Routing Load (NRL). It is found that the Exponential Traffic pattern gives better results as comparative to CBR Traffic pattern.
\end{abstract}

Keywords: AODV, DSR, Exponential Traffic, MANET, Manhattan Grid, NRL, PDR.

\section{INTRODUCTION}

A Mobile Ad-Hoc Network (MANET) is a self-configuring network of mobile nodes connected by wireless links, to form an arbitrary topology. The nodes are free to move randomly. Thus the network's wireless topology may be unpredictable and may change rapidly. Each node in the MANET is equipped with a wireless transmitter and receiver, with the aid of which it communicates with the other nodes in its wireless vicinity. The nodes which are not in wireless vicinity, communicate with each other hop by hop following a set of rules (routing protocol) for the hopping sequence to be followed. Routing protocols will need to perform four important functions of determination of network topology, maintaining network connectivity, transmission scheduling and channel assignment, and packet routing [1, 2]. Minimal configuration, quick deployment and absence of a central governing authority make ad hoc networks suitable for emergency situations like natural disasters, military conflicts, emergency and rescue operations, field applications etc. Each of these applications can potentially involve in different scenarios with different mobility patterns, traffic rates dependent on the environment and the nature of the interactions among the participants. In order to thoroughly study the protocols for these applications, it is imperative to use the mobility models that accurately represent the mobile nodes which utilize the protocols. In this paper, it is proposed to analyze the performances of two different routing protocols Ad-Hoc On-Demand Distance Vector Routing Protocol (AODV) and Dynamic Source Routing Protocol (DSR) against Manhattan Grid mobility model [3] in different traffic patterns: CBR and an Exponential traffic pattern with different mobility speed. We used the Exponential Traffic
Pattern which is very useful for generating multimedia traffic such as audio, video and text traffic etc [4].

Section 2 Reviews the Related work. Section 3 contains the description of Routing Protocols. Section 4 summarizes the Mobility model. Section 5 contains the overview of Traffic Patterns that considered in this paper. Section 6 includes the simulation Setup followed by results. Finally, concluding remarks are given in section 7 .

\section{RELATED WORK}

An extensive literature survey has been done to analyze the performance of routing protocols for various mobility models. Few researchers have carried out experiments to study the performance of reactive routing protocols such as DSR and AODV in mobile environments [5]. The traffic patterns perform an important role in the performance of routing protocols. Most researchers for a long time used the CBR traffic pattern for traditional data applications because of its simplicity and they used the different mobility models with CBR traffic pattern in different routing protocols such as: AODV, DSR and Destination Sequenced Distance Vector (DSDV). The protocols were mainly evaluated for packet delivery ratio and routing overhead. Recently, multimedia applications have drawn the attention of researchers a lot in MANET. These multimedia applications have a radically different traffic pattern. The data rate in voice application increases till it reaches a maximum peak. This pattern of traffic can be captured by Exponential distribution.

However, in the literature very few attempts were made to compare the performance analysis of MANET routing 
protocols in different traffic patterns. Thus, in this work, we intend to study the performance comparison of both CBR and Exponential traffic patterns in two different routing protocols with Manhattan Grid mobility model.

\section{III.Description OF Routing Protocols}

In an ad-hoc network, mobile nodes communicate with each other using multi hop wireless links. There is no stationary infrastructure; each node in the network also acts as a router, forwarding data packets for other nodes. Routing is the process of selecting paths in a network along which data to be sent. MANET routing protocols can be classified into three types [6]:

Proactive or table-driven protocols attempt to maintain consistent up-to-date routing information from each node to every other node in the network. Each node maintains tables to store routing information, and any changes in network topology need to be reflected by propagating updates throughout the network.

Reactive or on demand protocols are based on sourceinitiated on-demand reactive routing. If a node wants to send a packet to another node then this protocol searches for the route in an on-demand manner and establishes the connection in order to transmit and receive the packet [7]. Then, it initiates a route discovery process, which ends when the route is found.

Hybrid protocols combine the merits of proactive and reactive routing protocols.

\section{A. Ad-hoc On-demand Distance Vector Routing protocol $(A O D V)$}

The Ad-hoc On-Demand Distance Vector (AODV) routing protocol builds on the DSDV algorithm, it is an on demand routing algorithm, but in contrast to DSR it is a not source based routing scheme rather every hop of a route maintains the next hop information by its own [2]. Operation of the protocol here is also divided in two functions, route discovery and route maintenance. At first all the nodes send Hello message on its interface and receive Hello messages from its neighbours. This process repeats periodically to determine neighbour connectivity. When a route is needed to some destination, the protocol starts route discovery. The source sends Route Request Message to its neighbours [8]. If a neighbour has no information on the destination, it will send message to all of its neighbors and so on. Each receiving neighbour checks its routing table to see if it has a route to the destination. If it doesn't have a route to this destination, it will re-broadcast the RREQ packet and let it propagate to other neighbours. If the receiving node is the destination or has the route to the destination, a route reply (RREP) packet will be sent back to the source node. Routing entries for the destination node are created in each intermediate node on the way RREP packet propagates back. A hello message is a local advertisement for the continued presence of the node. Neighbours that are using routes through the broadcasting node will continue to mark the routes as valid. If hello messages from a particular node stop coming, the neighbour can assume that the node has moved away. When that happens, the neighbours will mark the link to the node as broken and may trigger a notification to some of its neighbours telling that the link is broken. In AODV, each router maintains route table entries with the destination IP address, destination sequence number, hop count, next hop ID and lifetime. Data traffic is then routed according to the information provided by these entries [9].

B. Dynamic Source Routing protocol (DSR)

The Dynamic Source Routing (DSR) protocol is an ondemand reactive routing protocol that is based on the concept of source routing. Operation of DSR can be divided in two functions, route discovery and route maintenance [10].

Route Discovery: When a source node $S$ wishes to send a packet to the destination node $D$, it obtains a route to $D$. This is called Route Discovery. Route Discovery is used only when $S$ attempts to send a packet to $D$ and has no information on a route to $D$.

- Route Maintenance: When there is a change in the network topology, the existing routes can no longer be used. In such a scenario, the source $\mathrm{S}$ can use an alternative route to the destination $D$, if it knows one, or invoke Route Discovery. This is called Route Maintenance.

The node floods the network with a route-request and builds the required route from the responses it receives. DSR allows the network to be completely self-configuring without the need for any existing network infrastructure or administration. The DSR protocol is composed of two main mechanisms that work together to allow the discovery and maintenance of source routes in the ad hoc network. All aspects of protocol operate entirely ondemand allowing routing packet overhead of DSR to scale up automatically [11].

\section{MoBILITY MODEL}

A mobility model should attempt to emulate the movements of real mobile nodes. Mobility models are based on setting out different parameters related to node movement. Basic parameters are the starting location of mobile nodes, their movement direction, velocity range, speed changes over time. There are many mobility models proposed for use in MANET [12]. Out of the several mobility models, in this work, we consider Manhattan Grid mobility model that is designed to capture a wide range of mobility patterns for ad-hoc applications.

\section{Manhattan Grid Mobility Model (MG)}

The random way point and RPGM models are the random mobility models where the movement of mobile nodes is freely moving at any direction. Manhattan Grid model has originally been developed to emulate the Manhattan street network, i.e. a city section which is only crossed by vertical and horizontal streets [13]. In some mobile applications, the movement of mobile nodes follows the mobility pattern similar to the road maps. In this model maps are used for the movement patterns. The map is composed of a number of horizontal and vertical streets. Each street has two lanes for each direction (North and South direction for vertical streets, East and West for 
horizontal streets). The mobile node is allowed to move along the grid of horizontal and vertical streets on the map. At an intersection of a horizontal \& vertical street, the mobile node can turn left, right or go straight [12]. The trajectories of mobile nodes are confined to a grid topology.

\section{Traffic Patterns}

\section{Constant Bit Rate (CBR) Traffic Pattern}

This traffic pattern generates data packets at a constant rate is very well known traffic model in the area of ad-hoc network. It is the most popular traffic source in network simulation. In this traffic, the data rate remains constant during the packet transmission [14]. It is good enough for the text transmission but this type of traffic pattern is not good so for multimedia applications.

\section{Exponential Traffic Pattern}

This traffic pattern have the specific features of multimedia applications and useful for the simulation of real time multimedia services. It follows an ON/OFF packet generation pattern. This traffic pattern is followed by an Exponential distribution and the packet generation rate varies following exponential distribution, during the ON period [4].

\section{VI.Simulation RESUlts AND ANALYSIS}

We use Bonn-Motion tool for mobility scenario generation [15]. We have taken 60 mobile nodes and Manhattan Grid mobility model. This section presents comparison and performance analysis of the two different routing protocols in various traffic patterns on the basis of Packet Delivery Ratio (PDR), Normalized Routing Load (NRL) and Throughput.

- Packet Delivery Ratio is the ratio of the number of packets originated by the application layer sources to the number of packets successfully delivered to their sink at the final destination.

- Normalized Routing Load is the number of control packets transmitted per data packet received at the destination.

- Throughput is the average rate of successful message delivery over a communication channel. This data may be delivered over a physical or logical link, or pass through a certain network node. The throughput is usually measured in bits per second (bit/s or bps), and sometimes in data packets per second or data packets per time slot.

TABLE I

SIMULATION PARAMETERS

\begin{tabular}{|l|l|}
\hline PARAMETER & VALUE \\
\hline Area size of topography $\mathrm{x}(\mathrm{m})$ & $1000 \mathrm{~m}$ \\
\hline Area size of topography y $(\mathrm{m})$ & $1000 \mathrm{~m}$ \\
\hline Mobility Model & Manhattan Grid \\
\hline Mobility Speed & $10-30-50 \mathrm{~m} / \mathrm{s}$ \\
\hline Number of Nodes & 60 \\
\hline Pause Time & 10 \\
\hline Simulation duration & $100 \mathrm{~s}$ \\
\hline Simulated Routing Protocols & AODV-DSR \\
\hline Transmission Range & $250 \mathrm{~m}$ \\
\hline Traffic Type & Cbr-Exponential \\
\hline
\end{tabular}

Impact of speed in MG Mobility Model of AODV

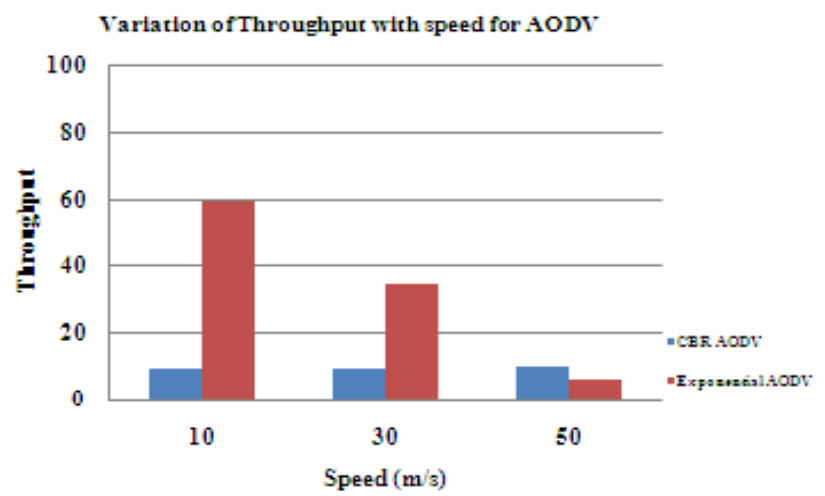

Fig.1 Throughput of MG in AODV Routing Protocol at different node speed on different traffic patterns

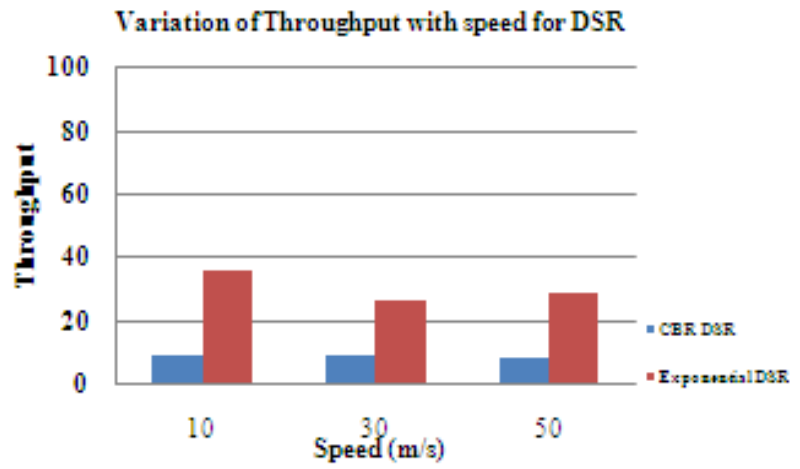

Fig.2 Throughput of MG in DSR Routing Protocol at different node speed on different traffic patterns

Variation ofPDR with speed forAODV

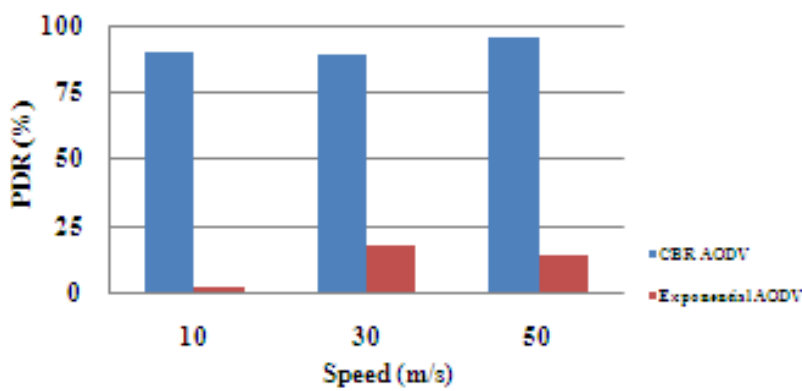

Fig.3 PDR of MG in AODV Routing Protocol at different node speed on different traffic patterns

Variation ofPDR with speed for DSR

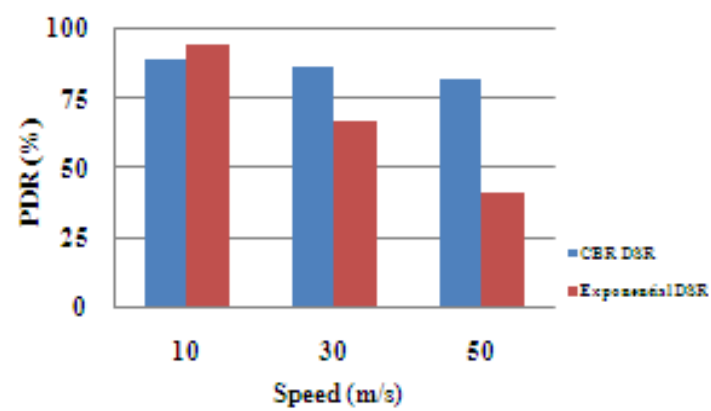


Fig.4 PDR of MG in DSR Routing Protocol at different node speed on different traffic patterns.

\section{Variation of NRL with speed for AODV}

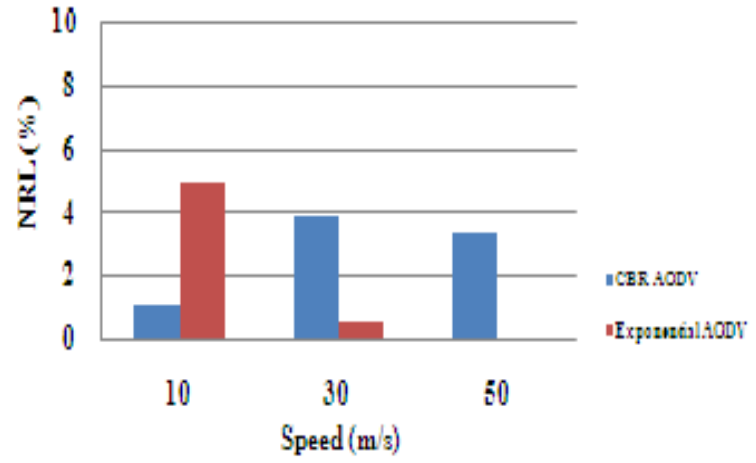

Fig.5 NRL of MG in AODV Routing Protocol at different node speed on different traffic patterns

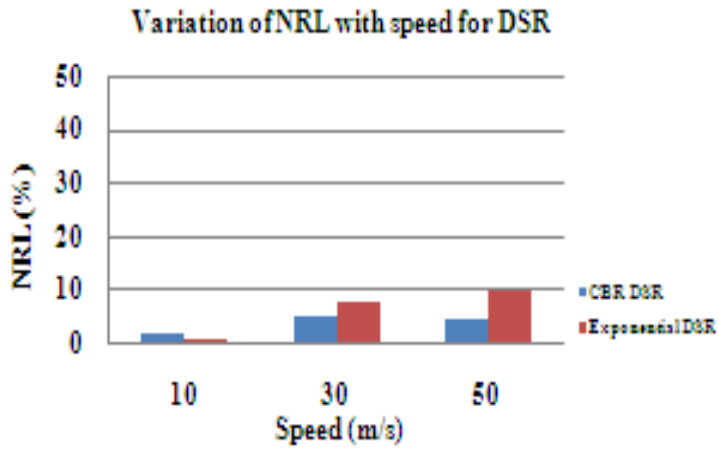

Fig.6 NRL of MG in DSR Routing Protocol at different node speed on different traffic patterns

The MG mobility model is map based mobility model and the Throughput for different traffic patterns with respect to different speed of the mobile nodes is very high in AODV as compared to DSR as can be seen in Fig.1 and Fig.2. In these figures throughput of Exponential traffic pattern is very high as compared to CBR traffic in both the routing protocols. The PDR of DSR routing protocols as shown in Fig.4 is good in exponential traffic pattern. NRL of DSR routing protocol as shown in Fig. 6 is high in exponential traffic pattern.

\section{CONCLUSION}

Empirical results illustrate that the performance of a Exponential traffic pattern in Manhattan Grid mobility model is better than the CBR traffic pattern in two different routing protocols Ad-Hoc On-Demand Distance Vector Routing Protocol (AODV) and Dynamic Source Routing Protocol (DSR) of Mobile Ad-Hoc Network with respect to different speed of the mobile nodes. We found that the AODV gives better results in throughput with exponential traffic pattern and DSR gives good results in PDR and NRL with exponential traffic pattern.

\section{REFERENCES}

[1] Gursharan singh, Sandeep Kaushal, Harpal "Comparative Analysis of Stable Link Management for Improvement of Quality of Service in Manets", International Journal of Advanced Research in Computer Science and Software Engineering Vol.4, Issue 9, pp 180-183, September 2014

Copyright to IJARCCE
[2] Royer E.M.and Toh C., "A review of current routing protocols for adhoc mobile wireless networks", IEEE personal communications, 1999, pp. 46-56.

[3] T. Camp, J. Boleng and V. Davies, "A Survey of Mobility Models for Ad Hoc Network Research," in the proceedings of Wireless Communications \& Mobile Computing (WCMC), 2002

[4] Arindrajit Pal, Jyoti Prakash Singh, Paramartha Dutta "The Effect of speed variation on different Traffic Patterns in Mobile Ad Hoc Network." Published by Elsevier Ltd. Selection and/or peer-review under responsibility of C3IT. Procedia Technology 42012 pp $743-$ 748 doi:10.1016/jprotcy.2012.05.121

[5] J. Broch, D. A. Maltz, D. B. Johnson, Y.-C. Hu and J. Jetcheva, “A performance comparison of multi-hop wireless ad hoc network routing protocols," in Proceedings of the Fourth Annual ACM/IEEE International Conference on Mobile Computing and Networking, ACM, October 1998.

[6] R. Mohan, C. Rajan and Dr. N. Shanthi "A Stable Mobility Model Evaluation Strategy for MANET Routing Protocols." International Journal of Advanced Research in Computer Science and Software Engineering, Volume 2, Issue 12, pp.58-65, December 2012

[7] Harpal, Sandeep Kaushal, Gursharan Singh "Comparison of Routing Protocols in MANET" Proceeding of PTU sponsored national conference on Latest Development in Science, Engineering \& Management (LDSEM), pp 263-268, March 2014

[8] Yasser Kamal Hassan, Mohamed Hashim Abd El-Aziz, and Ahmed Safwat Abd El-Radi "Performance Evaluation of Mobility Speed over MANET Routing Protocols." International Journal of Network Security, Vol.11, No.3, pp.128-138, Nov 2010

[9] Aditya Goel, Ajaii Sharma, "Performance Analysis of Mobile Adhoc Network Using AODV Protocol", International Journal of Computer Science and Security (IJCSS), Volume (3): Issue (5), Page No.334

[10] David B. Johnson, David A. Maltz, Yih-Chun Hu, The Dynamic Source Routing (DSR) Protocol for Mobile Ad Hoc Networks.draftietf-manet-dsr-10.txt, July 2004

[11] David B. Johnson and David A. Maltz. "Dynamic Source Routing in Ad Hoc Wireless Networks". In Mobile Computing, edited by Tomasz Imielinski and Hank Korth, Chapter 5, pages 153-181, Kluwer Academic Publishers, 1996.

[12] T. Camp, J. Boleng and V. Davies, "A Survey of Mobility Models for Ad Hoc Network Research," in the proceedings of Wireless Communications \& Mobile Computing (WCMC), 2002

[13] Valentina Timcenko, Mirjana Stojanovic and Slavica Bostjancic Rakas "MANET Routing Protocols vs. Mobility Models: Performance Analysis and Comparison." Proceedings of the 9th WSEAS International Conference on Applied Informatics and Communications (AIC'09), pp 271 - 276, Serbia, 2009.

[14] A. Al-Maashri, M. Ould-Khaoua, Performance analysis of manet routing protocols in the presence of self-similar traffic, in: $31^{\text {st }}$ IEEE Conference on Local Computer Networks, Tampa, Florida, USA, 2006, pp. 811-818

[15] University of Bonn, Bonnmotion - a mobility scenario generation and analysis tool, http://www.cs.unibonn.de/IV/bomonet/BonnMotion.htm 